\title{
Constitución de los Estados Unidos: Mutación de su Normativa
}

Constitution of the United States:

Mutation of the Constitutional Text

\section{Mgtr. Francisco López Rueda}

Profesor Titular PUCE (Facultad de Jurisprudencia)

Artículo Original (Investigación)

RFJ, No. 1, 2017, pp. 203-217, ISSN 2588-0837

RESUMEN: El artículo explica brevemente la teoría de la mutación constitucional y su diferencia con una reforma o una enmienda a una constitución. Al partir de esto, el artículo hace un estudio breve sobre mutaciones de la Constitución de los Estados Unidos y su influencia en la forma en la que el Derecho es entendido en este país, así como también la influencia de una mutación en sistemas jurídicos alrededor del mundo.

PALABRAS CLAVE: Mutación, Constitución, Corte Suprema de los Estados Unidos, Hermenéutica, Enmienda.

ABSTRACT: The article briefly explains the theory of a constitutional mutation, and the difference between it and a constitutional reform or a constitutional amendment. The article discusses the influence of the mutation of the Constitution of the United States among Law around the world. At the same time, the article provides an opinion about the influence of the mutation of the Constitution of the United States upon legal systems all worldwide.

KEYWORDS: Constitutional mutation, Constitution, Supreme Court of the United States, Hermeneutics, Amendment.

\section{INTRODUCCIÓN}

La mutación constitucional, como forma de alterar el contenido de una constitución, ha sido poco estudiada. Sin embargo, no es ajena a doctrinarios, especialmente europeos, que han tratado de explicarla 
como una forma válida de interpretación constitucional. La mutación -tal como su nombre lo indica- es el cambio, pero no un cambio físico, sino un cambio implícito, no expreso.

Todos los sistemas jurídicos alrededor del mundo han realizado mutaciones a sus constituciones, aun cuando no hayan caído en cuenta que las realizaron. El trabajo que se presenta a continuación tiene como objetivo explicar brevemente algunas mutaciones que ha sufrido la Constitución de los Estados Unidos sin que este documento hubiere sufrido alguna alteración en su texto, vigente desde 1789 .

Una mutación constitucional va más allá de una simple interpretación. Es la concreción de un cambio, un cambio en el sentido propio de la constitución. Las mutaciones constitucionales buscan darle un nuevo sentido al texto constitucional. En la mayoría de los casos este nuevo sentido busca refrescar un documento del cual depende no solo la estabilidad jurídica, sino también la estabilidad política de todo un país.

En las siguientes páginas se podrá leer un estudio del inicio de las mutaciones constitucionales, así como también mutaciones que ha sufrido la Constitución de los Estados Unidos en un tema en concreto: la cláusula de comercio del artículo 1 de la Norma Suprema.

\section{REFORMA Y MUTACIÓN CONSTITUCIONAL}

El valor de una constitución no se mide por su contenido o texto escrito, sino por la forma en la que las personas que la aplican aceptan el alcance de sus normas. En la correcta aplicación del texto constitucional, una analogía válida sería la de una prenda de vestir y el cuerpo del dueño de la prenda. La prenda debe amoldarse al cuerpo de la persona y no al contrario. Lo mismo sucede con una constitución y la sociedad en la que sus normas van a regir. No es la sociedad la que debe acoplarse a una constitución irreal, sino que es la constitución la que debe reflejar la realidad de la sociedad a la que va a regular. Al desarrollar la analogía, así como las personas nos encontramos en graves problemas a la hora de vestir una prenda más grande o más pequeña que nuestros cuerpos, una sociedad enfrenta complicaciones al tratar de acatar normas constitucionales alejadas de la realidad.

Es cuestionable entonces la posición de algunos autores, adeptos o adictos a corrientes constitucionales que han calado en países como 
Ecuador, Bolivia y Venezuela, principalmente, que sostienen que las constituciones están para traer nuevos paradigmas e ilusiones a las sociedades actuales en busca de un orden que se nutre de muchos otros componentes, no solo el jurídico. Una constitución que no refleja la realidad del país en el que va a regir es un texto condenado a sufrir de un interminable lirismo.

Sería sencillo copiar literalmente textos constitucionales que han resultado exitosos en otros países para así encontrar un equilibrio en sociedades que aun enfrentan retos a la hora de acatar normas básicas de convivencia. La historia nos ha demostrado que hacerlo no lleva a buen futuro a los países en los que los gobernantes creen que elaborar normas jurídicas perfectas -como si estas existiesen- pueda cambiar el destino de toda una nación.

América Latina ha sido el perfecto ejemplo de como la adopción de constituciones irreales provoca, no solo inestabilidad en todos los órdenes estatales, sino también el constante cambio y reforma de normas supremas en busca de un nuevo orden estatal. Concretamente, Ecuador desde su independencia en 1830 ha tenido un número elevadísimo de textos constitucionales, al llegar a los 20, es decir, en un promedio de una constitución nueva cada 10 años ${ }^{63}$

Ahora bien, creer que una constitución es un texto cuasi sagrado e inmutable también es poco realista. Una constitución no puede permanecer sin ningún cambio a través del tiempo, puesto que las sociedades también cambian. No hay mayor sentido en creer que mientras más intocable una constitución, mayor la seguridad jurídica de un país. La seguridad jurídica como tal no solo depende de la poca reforma de normas escritas, sino también de la estabilidad del orden jurídico con el pasar del tiempo.

Se presentan entonces, sin ser exclusivas, dos opciones cuando una constitución deja de ajustarse a la realidad de un país. Una reforma expresa de la constitución, esto es, activar el poder constituyente, o por otro lado, que el órgano encargado del control de constitucionalidad realice interpretaciones extensivas del texto constitucional que le permitan, sin alterar el sentido o el espíritu de la constitución,

63 Se puede consultar los textos de las constituciones de Ecuador en la página web de la Asamblea Nacional del Ecuador, en http://archivobiblioteca.asambleanacional. gob.ec/constituciones-del-ecuador. 
acoplar las normas constitucionales, en su tenor literal caducas, a una aplicación actual y flexible.

La primera opción, la reforma constitucional, es la solución más salomónica en el sentido de contar con varias opiniones dentro de la sociedad sin dejar de lado a actores que podrían aportar una visión distinta de la del constituyente, enquistado este último en tecnicismos jurídicos ajenos en algunos de los casos a la voluntad popular. La segunda opción es la interpretación del texto por parte del órgano encargado del control de constitucionalidad, y podría resultar riesgosa.

La reforma constitucional en estos casos daría mayor seguridad, puesto que el poder constituyente derivado entraña siempre el acatamiento de normas o reglas para su funcionamiento. La reforma constitucional no se activa sin el designio previo de las normas relativas al tema. Una constitución sigue un camino previamente trazado para su propio cambio.

La reforma constitucional, por su naturaleza misma, difiere de un mero cambio legal, lo cual da mayor estabilidad a una constitución. No es sano para una sociedad que una reforma se dé sin mayores dificultades, así como tampoco es sano tener una constitución extremadamente rígida.

Por su parte, la interpretación constitucional puede convertirse en riesgosa, puesto que esta depende no solo de técnicas legales y doctrinarias, sino básicamente de la visión de la persona que realiza el ejercicio interpretativo y de su posición política.

Una interpretación constitucional, sin límites claros, puede fácilmente caer en una situación que desvirtúe el sentido o el espíritu mismo de la norma suprema. Tal como Canosa (1998) explica, es la propia constitución la que da ciertas "pautas hermenéuticas" que deben ser acatadas por el intérprete para evitar salirse del sentido claro de la norma.

La cuestión está en saber si esas "pautas hermenéuticas" tienen un sentido más cercano a una regla de interpretación o si solamente son meros lineamientos que el intérprete no debería abandonar, pero que en sí no son una norma de estricto cumplimiento.

En caso de que las pautas sean meros lineamientos, el riesgo que se presenta en la interpretación es que esta sea tan libre y extensiva que 
esto no dependa de la norma como tal, sino de la visión del intérprete y esto comprende no solo su formación académica, sino también sus convicciones personales, sin las cuales su trabajo de interpretación de un texto constitucional no podría llegar a ser lo suficientemente válido.

Una interpretación libre y extensiva del texto constitucional bien podría terminar en una forma sui generis e innovadora de ver a la constitución lo cual beneficiaría a la sociedad entera, pero por otro lado podría devenir en un efecto poco deseado en sociedades respetuosas de la norma jurídica, esto es, en una mutación constitucional.

\subsection{Mutación constitucional}

Al hablar de una mutación constitucional, tal como su nombre lo indica, es un claro cambio en el sentido de la constitución de un país, pero este guarda pocas similitudes a una reforma constitucional. Una reforma cambia el sentido literal del texto para futuro, al hacer que el intérprete deba ajustarse a una nueva forma de entender al pasaje como tal.

En la mutación constitucional nos encontramos frente a un cambio del sentido del texto de la constitución, sin que este cambie expresamente. La mutación depende del órgano que interpretó la constitución, lo cual puede tornarse en extremadamente subjetivo al tener en cuenta que, a simple lectura, la constitución resulta la misma que el estudiante, el abogado o el lector común pueda observar o estudiar, pero el sentido en el que es aplicada ha cambiado.

Al considerar en cuenta lo anterior, la mutación constitucional está íntimamente ligada a la interpretación del texto de la norma suprema. Una mutación ahorra a la persona o grupo de personas que la interpretan el proceso, en algunos casos lento y demorado, de reformar la constitución bajo sus propios procedimientos.

Amparados en el concepto de mutación constitucional se puede opinar que la constitución goza de una cierta estabilidad, puesto que no es reformada expresamente y su texto como tal sigue indemne, pero el sentido de su texto ha cambiado, de forma tal que si ese hubiese sido objeto de una reforma, tal vez hubiese requerido no solo el cambio de uno o varios artículos en específico, sino también la adopción de una nueva constitución para un país. 
En cierto sentido esta institución, la mutación, respeta principios de perdurabilidad en el tiempo de una constitución. Sin alterar procesos formales de reforma, o sin caer en acciones inconstitucionales, la mutación ofrece la posibilidad de entender al texto de una forma distinta y nueva que se ajuste a una constitución vieja a tiempos modernos.

La mutación constitucional como doctrina tiene una vida relativamente reciente. Parte de una conferencia de Georg Jellinek pronunciada en 1908, la que posteriormente traducida y adaptada al castellano por Manuel García Pelayo adquiere el nombre de mutación.

Posterior a los trabajos de Jellinek, varios autores como Hsü-DauLin y Heller desarrollaron bajo sus propias perspectivas a la doctrina de mutación constitucional (Da Silva, 1999). La mutación como tal tiene una finalidad clara: flexibilizar textos constitucionales que en sentido formal gocen de una rigidez incuestionable. La doctrina se desarrolla en Europa en un período entre guerras, tiempo en el cual los cambios en todo el Derecho Constitucional fueron de especial importancia para el destino del planeta entero.

Conrad Hesse, más conservador en cuanto a la mutación constitucional, opinaba que una mutación sería válida si y solo si esta no cambiaba el sentido del texto constitucional. Para Hesse (Da Silva, 1999) las mutaciones constitucionales dejan de tener sentido cuando la variación de la constitución es tal que la misma debilita como tal a la norma suprema, desproveyéndola de la estabilidad que necesita para ser la norma madre del ordenamiento jurídico.

Bajo este razonamiento, las mutaciones constitucionales solo serían válidas, en una perspectiva absolutamente conservadora, para solventar o llenar vacíos constitucionales. En este sentido se puede hablar de una "función constituyente difusa" mediante la cual la mutación constitucional solo complementa el trabajo que el constituyente omitió, bien sea por descuido, bien por una intencionalidad clara en la que el constituyente considera que es mejor dejar cabos sueltos en una constitución para que esta sea interpretada por el órgano de control de constitucionalidad, en muchos casos las cortes de justicia, así como también las cortes o tribunales constitucionales.

Otros autores como Parejo (2005) también tiene la misma postura al sostener que una mutación constitucional no puede llenar lagunas 
o vacíos constitucionales, puesto que esto indefectiblemente derivará en una infracción constitucional.

En relación a la opinión de Hesse, ¿Cuál sería el sentido de una mutación constitucional, sin que esta varíe el sentido de la constitución? No hay sentido alguno en poner en práctica una mutación que no cambie el sentido del texto de la norma. Una mutación que únicamente llene vacíos constitucionales no es propiamente una mutación, sino solamente una interpretación técnica.

La mutación constitucional como tal es una institución que está en constante desarrollo. Es difícil determinar de dónde surge una mutación y cuáles son sus límites, así como llegar a determinar una mutación válida de una que solamente sea una inconstitucionalidad flagrante y en fiel contravención al espíritu de la constitución entera.

"Hsü-Dan-Lin distingue cuatro tipos de mutaciones constitucionales: a) las debidas a prácticas políticas que no se opongan formalmente a la Constitución escrita, y para cuya reglamentación no exista norma constitucional; b) las debidas a prácticas políticas en oposición abierta a preceptos constitucionales; c) las producidas por imposibilidad del ejercicio, o por desuso, de las competencias y atribuciones establecidas en la Constitución; d) las producidas por interpretación de los términos de la Constitución, de tal modo que los preceptos obtienen un contenido distinto de aquel con el que en principio fueron pensados"(Da Silva, 1999) ${ }^{64}$.

Para el objetivo de este trabajo, es decir, analizar las mutaciones de la Constitución de los Estados Unidos, al partir de la clasificación que Hsü-Dan-Lin realizó, se partirá por analizarlas desde el punto d del parágrafo anterior. Las mutaciones en Estados Unidos, más que por motivos políticos, se han dado para dotar al texto de norma suprema de un contenido distinto de aquel que se puede leer. Aun con la influencia política de los miembros de la Corte Suprema de Estados Unidos, estos a través de su historia, han desarrollado mutaciones con un sentido más técnico jurídico que político.

64 En el trabajo del profesor José Alfonso Da Silva no se establece una referencia a la traducción oficial de los ensayos de Hsü-Dan-Lin, por lo que se utiliza una traducción e interpretación de Pedro de la Vega. 


\subsection{Mutación de la Constitución de los Estados Unidos}

El mito bajo el cual el mundo del Derecho vive y ha vivido es que la Constitución de los Estados Unidos es uno de los textos fundamentales más estables del mundo, además de la Constitución de un estado federal con más antigüedad. Al tener en cuenta que es uno de los textos constitucionales más pequeños del mundo, con solo 4400 palabras, esta norma ha sido el conducto de grandes cambios en el mundo jurídico, no solo en Estados Unidos, sino también en el resto del mundo.

Irónico resulta decir que un texto tan pequeño pueda regular tantos temas a la vez. Es necesario decir que esto también se da por la forma en la que el sistema jurídico del case law funciona, distinto del sistema civilista. El sistema jurídico que se maneja en Estados Unidos -que deberá ser objeto de un trabajo distinto y más extenso- se regula por precedentes jurisprudenciales más que en sí por la norma escrita, tal como en el sistema civilista.

A lo largo de sus 228 años de vida, la Constitución de Estados Unidos nunca ha sido reformada, y solo ha tenido 27 enmiendas, al representar la última aprobada en 1992. Incluso su procedimiento de reforma, regulado en el artículo 5 de la norma suprema, a pesar de su aparente sencillez, tan distinto a los procesos de reforma en América Latina, entraña una complejidad absoluta.

El proceso de enmienda de la Constitución se puede dar solamente por la aprobación de ambas cámaras del Congreso de Estados Unidos, así como también por las legislaturas de los 50 estados, es decir, que debe existir un consenso generalizado que el cambio político que va a tener el país por medio de la enmienda debe favorecer a la Unión en general, no solo a alguno o a un grupo de estados. Por este motivo, como ya se ha señalado, la Constitución ha tenido solamente 27 enmiendas en temas fundamentales para el país entero.

Por otra parte, se puede convocar a una convención nacional para que proponga enmiendas a la Constitución; huelga decir que este procedimiento nunca se ha puesto en marcha en 228 años. Se podría llegar a considerar que esta convención nacional es lo más parecido a una asamblea constituyente en países latinoamericanos. 
La pregunta que cabe con estos antecedentes es si la Constitución de Estados Unidos no ha tenido una reforma a lo largo de su vida jurídica, ni ha tenido demasiadas enmiendas, ¿̇cómo ha logrado sobrevivir en el tiempo?

La respuesta no es sencilla y deberá ser analizada en un trabajo mucho más amplio, pero para efectos de este artículo, luego de una reflexión del autor, la conclusión es que la Constitución de los Estados Unidos ha pasado por varios procesos de mutación por parte de la Corte Suprema del país. Ha sido el análisis de casos particulares el que lleva a la interpretación extensiva del texto normativo de la Constitución.

La interpretación por parte de los jueces de la Corte Suprema se hace, en apariencia, libre de cualquier vinculación política, aunque no en todos los casos; pero esto ha ocasionado que algunos apartados de la Constitución pierdan su sentido original y, a su vez, tengan un alcance normativo mucho mayor del que su texto dicta.

La técnica de interpretación de la Constitución, lo cual ha facilitado su mutación, depende del juez que la estudie. No todos los jueces de la Corte Suprema de Estados Unidos tienen la misma visión del alcance del texto constitucional, pero todos han interpretado normas en aplicación directa de casos individuales. Varios de estos casos son los que han activado la mutación constitucional.

Existe un tema en específico en la Constitución de los Estados Unidos que ha sido objeto de una interpretación tal por parte de los jueces de la Corte Suprema, que se considera como una mutación constitucional. Este es el caso de la commerce clause.

El artículo 1 de la Constitución de los Estados Unidos regula a la función legislativa, al Congreso y sus dos cámaras; este artículo, dividido en secciones, enumera las atribuciones del legislativo. Entre las atribuciones del Congreso está la de regular el comercio con otras naciones, entre estados de la federación y con las tribus indígenas.

"The Congress shall have the power... (to) regulate Commerce with foreign Nations, and among several States, and with the Indian Tribes..." ${ }^{65}$

Estas dos -en apariencia- simples líneas le dan poder al Congreso de los Estados Unidos sobre un aspecto de fundamental importancia

65 Artículo 1 §8 numeral 3 de la Constitución de los Estados Unidos. 
para el país, el comercio. A simple lectura no hay ningún aspecto extra al comercio que este apartado garantice o norme, pero con el paso del tiempo la Corte Suprema, a través de este artículo, ha llegado al extremo de garantizar derechos fundamentales.

La regulación del comercio, acorde a la lectura de ese artículo, es el punto central de ese apartado, pero llegar a pensar en garantía de derechos fundamentales no tiene otra explicación clara que una mutación constitucional. A continuación, se explicarán dos casos en los que la interpretación de los jueces desembocó en una mutación sin alterar el tenor literal del texto constitucional.

El desarrollo jurisprudencial, propio del case law system, sería de gran ayuda para desarrollar una variación del sentido de la Constitución sin caer en una reforma expresa o en una enmienda innecesaria para el texto constitucional. La sola interpretación del alcance de la norma es suficiente para dar un ámbito de protección a una sección que ordinariamente no toparía ningún tema relativo a derechos fundamentales.

El contexto histórico en el que se desarrollan las dos sentencias que operativizan la mutación constitucional se sitúa en la segunda mitad del siglo XX, concretamente con la Ley de Derechos Civiles de 1964, la cual frenó la discriminación y segregación en Estados Unidos, por lo menos en apariencia. La Ley de Derechos Civiles establecía que era ilegal que "any inn, hotel, motel, or other establishment which provides lodging for transient guests to discriminate on the basis of race, color, religion, or national origin" (May, 2013).

Las dos sentencias a las que se ha hecho alusión son Heart of Atlanta Motel, Inc. v. United States (1964) y Katzenbach v. McClung (1964). En el primer caso, el dueño de un motel, el Heart of Atlanta, que tenía 216 habitaciones, gozaba de una ubicación privilegiada en Atlanta y según sus registros el 75\% de sus huéspedes eran de fuera del Estado (Chemerinsky, 2015), decidió demandar la inconstitucionalidad de la Ley de Derechos Civiles, puesto que consideró que la misma atentaba contra la commerce clause al prácticamente obligarle aceptar en sus instalaciones a todo tipo de huéspedes, incluidos afroamericanos.

La Corte, en su interpretación de la Constitución, decidió que las acciones del dueño del Heart of Atlanta tenían una afectación para el comercio entre estados, ya que, si el $75 \%$ de huéspedes provenían de fuera del Estado, entonces la discriminación o la no aceptación de 
afroamericanos en su establecimiento era un tema que, fuera de la vulneración de derechos civiles, afectaba más que nada al comercio entre estados, lo cual debía ser regulado por el Congreso. La Corte afirmó que "voluminous testimony (before Congress) presents overwhelming evidence that discrimination by hotels and motels impedes interstate travel" (Chemerinsky, 2015).

La mutación de la Constitución es clara. La Corte con su interpretación le dio un nuevo sentido a la sección de la cláusula de comercio en el texto constitucional, ya que en el tenor literal no se regula absolutamente nada sobre derechos civiles, dado que todo es un tema netamente económico, pero la Corte decidió que este aspecto económico acabaría por proteger derechos civiles. El texto escrito ha permanecido intacto, sin embargo, el alcance de su interpretación cambió y ha servido como precedente para poder proteger derechos civiles en casos en los que el comercio se vea envuelto.

Por otro lado, en Katzenbach v. McClung, un restaurante familiar, el Ollie's Barbecue, situado en Birmingham, Alabama, bajo el mismo criterio del Heart of Atlanta afectó derechos civiles en estricta relación con el comercio entre estados. En este caso, el Ollie's Barbecue utilizaba al año un $46 \%$ de carne proveniente de fuera del Estado de Alabama, con lo que la decisión del dueño del restaurante de no aceptar a afroamericanos en su local afectaba directamente el comercio entre estados, con lo cual los derechos civiles nuevamente se vieron afectados por una relación de ámbitos comerciales.

La Corte, para fortalecer sus argumentos, empleó testimonios que se llevaron a cabo en el Congreso de Estados Unidos mientras se elaboraba la Ley de Derecho Civiles; es así que según estos testimonios "afforded ample basis for the conclusion that established restaurants in such areas sold less interstate goods because of the discrimination that interstate travel was obstructed directly by it, that business in general suffered and that many new businesses refrained from establishing there as a result of it" (Chemerinsky, 2015).

En este caso la Corte, como ya se ha dicho, llegó a la conclusión que la vulneración de derechos de afroamericanos afectaba directamente el comercio entre estados. Esto no solo es una interpretación de la Constitución, sino que también es una mutación de la misma. Es difícil encontrar precedentes en los cuales derechos civiles o fundamentales sean protegidos a través del comercio entre estados o países. 
Es necesario destacar que los miembros de la Corte Suprema en ambos casos tomaron las decisiones de forma unánime. A través de su interpretación libre, los jueces de la Corte mutaron el contenido de la Constitución. En un hábil y conveniente movimiento, manejaron el momento político con brillantez. El Judicial era el único poder que faltaba por respaldar a la Ley de Derechos Civiles presentada por el Ejecutivo y aprobada por el Legislativo, con lo cual la mutación constitucional dio el toque final de legitimidad a la nueva visión del país para la protección de un grupo humano largamente discriminado.

La mutación en este par de casos en Estados Unidos no cambió el texto constitucional, no reformó la Constitución expresa o tácitamente. El texto original de 1789 es el mismo, pero su interpretación ya no es la misma. Bajo cualquier consideración la cláusula de comercio de la Constitución de Estados Unidos, a simple lectura, no es más que regular aspectos comerciales o mercantiles del país, pero a partir de 1964 esto ya no es así. La cláusula de comercio, por interpretación del órgano de control de constitucionalidad, pasó a garantizar derechos fundamentales o civiles.

Bajo la misma perspectiva, aunque es un tema de otro artículo por su amplitud, la Constitución de los Estados Unidos sufrió una de sus primeras mutaciones con una decisión de la Corte Suprema que ha sido la piedra angular de sistemas jurídicos alrededor del mundo desde 1803, Marbury v. Madison. En esta decisión, que fue el fundamento para el judicial review o control de constitucionalidad, la Corte mutó de tal forma el contenido de la Constitución, que esta le dio poderes amplios a la Corte Suprema que el soberano, esto es, el pueblo, no le dio en el texto original de la Constitución.

La sentencia Marbury v. Madison fue fruto de una interpretación extremadamente extensiva por parte de la Corte. Por su importancia, pocas han sido las voces que se han atrevido a criticarla o contradecirla (Sanín, 2014). Sin embargo, fue un logro descomunal de miembros de un ala política que se aseguraron controlar un poder del Estado mientras perdieron los otros dos. Marbury v. Madison no nació por una necesidad jurídica, sino por una necesidad política de controlar el poder.

La Constitución de los Estados Unidos ha mutado, sin necesidad de que sea reformada, desde pocos años de su nacimiento. Una mutación constitucional influyó en la forma de entender el Derecho Constitucional alrededor del mundo desde mucho antes que la idea de muta- 
ciones constitucionales exista. En 1803 una mutación constitucional cambió el destino de casi todos los sistemas jurídicos.

La sentencia a la que se ha hecho alusión dio un poder gigante a un órgano de control de constitucionalidad que no tiene ese poder en el texto escrito de la Constitución. La propia Constitución no les reconoce ese poder a los jueces, sin embargo, estos ejercen ese poder inconmensurable a diario, fruto de una mutación constitucional.

Para concluir, la mutación constitucional es una herramienta útil para controlar la forma en la que se aplica una constitución, pero esto depende no solo de fundamentos técnicos, sino también éticos. Una mutación constitucional con fundamentos poco éticos puede llegar a desvirtuar al espíritu de la norma suprema sin que un órgano político pueda frenarlo, ya que es casi imposible frenar una variación en el contenido de la constitución que no se haga de forma expresa. Intentar frenar una mutación constitucional conllevaría a entrometerse en el trabajo de una de las funciones del Estado, la Judicial, sin tener una justificación clara. En pocas palabras, frenar una mutación constitucional requeriría de un desequilibrio en los poderes del Estado.

\section{REFERENCIAS BIBLIOGRÁFICAS}

\section{Libros}

Balaguer Callejón, M. L. (1997). Interpretación de la Constitución y ordenamiento jurídico. Madrid. Editorial Tecnos.

Canosa Usera, R. (1988). Interpretación constitucional y fórmula política. Madrid. CEC.

Chemerinsky, E. (2015). Constitutional Law principles and policies. New York. Wolters Kluwer.

De Cabo Martín, C. (2003). La Reforma constitucional. Madrid. Editorial Trotta.

Gargarella, R. (2011). La Constitución en 2020. Argentina. Siglo Veintiuno Editores. 
May, C. (2013). Constitutional Law individual rights. New York. Wolters Kluwer.

Ramírez Cleves, G. (2003). Los límites a la reforma constitucional y las garantías - límites del poder constituyente: los derechos fundamentales como paradigma. Bogotá. Universidad Externado de Colombia.

Rodríguez Garavito, C. (2011). El derecho en América Latina. Argentina. Siglo Veintiuno Editores.

Sanín Restrepo, R. (2014). Teoría crítica constitucional. Valencia. Tirant lo Blanch.

Schmitt, C. (2003). Teoría de la Constitución. Madrid. Alianza Universidad Textos.

\section{Capítulos de libro}

Parejo Alfonso, L. (2005). Las mutaciones constitucionales. Algunos ejemplos extraídos de la experiencia española y europea. En varios autores (2005). Reforma de la constitución y control de constitucionalidad. Bogotá. Pontificia Universidad Javeriana. Facultad de Ciencias Jurídicas.

\section{Artículo de la web}

Da Silva, J. A. (1999). Mutaciones constitucionales. Recuperado de: https://revistas.juridicas.unam.mx/index.php/cuestionesconstitucionales/article/view/55617 
Recibido: 10/02/2017

Aprobado para publicación: 27/05/2017

Francisco López Rueda (LLB, LLM): Es abogado por la UDLA, Especialista Superior en Derecho Constitucional por la UASB y Master of Laws (LLM) por la University of Southern California. Ha sido funcionario de la ONU, así como también Asesor en la Asamblea Nacional del Ecuador, Coordinador Jurídico en el CEAACES y Director de Regulación en el CORDICOM. Su práctica privada gira en torno a la asesoría empresarial y laboral. En la actualidad se desempeña como docente docente titular de la PUCE.

Correo electrónico: FJLOPEZ@puce.edu.ec 